\title{
Congruences modulo powers of 5 for the rank parity function
}

\author{
Dandan Chen, Rong Chen and Frank Garvan
}

\begin{abstract}
It is well known that Ramanujan conjectured congruences modulo powers of 5, 7 and 11 for the partition function. These were subsequently proved by Watson (1938) and Atkin (1967). In 2009 Choi, Kang, and Lovejoy proved congruences modulo powers of 5 for the crank parity function. The generating function for the rank parity function is $f(q)$, which is the first example of a mock theta function that Ramanujan mentioned in his last letter to Hardy. We prove congruences modulo powers of 5 for the rank parity function.
\end{abstract}

Keywords. partition congruences, Dyson's rank, mock theta functions, modular functions

2010 Mathematics Subject Classification. 05A17, 11F30, 11F37, 11P82, 11P83

\section{Introduction}

Let $p(n)$ be the number of unrestricted partitions of $n$. Ramanujan discovered and later proved that

$$
\begin{aligned}
p(5 n+4) & \equiv 0 \quad(\bmod 5) \\
p(7 n+5) & \equiv 0 \quad(\bmod 7) \\
p(11 n+6) & \equiv 0 \quad(\bmod 11)
\end{aligned}
$$

In 1944 Dyson [Dy44] defined the rank of a partition as the largest part minus the number of parts and conjectured that the residue of the rank mod 5 (resp. mod 7) divides the partitions of $5 n+4$ (resp. $7 n+5$ ) into 5 (resp. 7) equal classes thus giving combinatorial explanations of Ramanujan's partition congruences mod 5 (resp. 7). Dyson's rank conjectures were proved by Atkin and Swinnerton-Dyer [AS-D54]. Dyson also conjectured the existence of another statistic, he called the crank, which would likewise explain Ramanujan's partition congruence mod 11. The crank was found by Andrews and the third author [AnGa88] who defined the crank as the largest part, if the partition has no ones, and otherwise as the difference between the number of parts larger than the number of ones and the number of ones.

Let $M_{e}(n)$ (resp. $\left.M_{o}(n)\right)$ denote the number of partitions of $n$ with even (resp. odd) crank. Choi, Kang and Lovejoy [CKL09] proved congruences modulo powers of 5 for the difference, which we call the crank parity function.

Theorem 1.1. (Choi, Kang and Lovejoy [CKL09, Theorem 1.1]) For all $\alpha \geq 0$ we have

$$
M_{e}(n)-M_{o}(n) \equiv 0 \quad\left(\bmod 5^{\alpha+1}\right), \quad \text { if } 24 n \equiv 1 \quad\left(\bmod 5^{2 \alpha+1}\right) .
$$

This gives a weak refinement of Ramanujan's partition congruence modulo powers of 5 :

$$
p(n) \equiv 0 \quad\left(\bmod 5^{\alpha}\right), \quad \text { if } 24 n \equiv 1 \quad\left(\bmod 5^{\alpha}\right) .
$$

This was proved by Watson [Wa38].

The first and second authors were supported in part by the National Natural Science Foundation of China (Grant No. 11971173). The third author was supported in part by a grant from the Simon's Foundation (\#318714).

We thank episciences.org for providing open access hosting of the electronic journal Hardy-Ramanujan Journal. 
In this paper we prove an analogue of Theorem 1.1 for the rank parity function. Analogous to $M_{e}(n)$ and $M_{o}(n)$, we let $N_{e}(n)$ (resp. $N_{o}(n)$ ) denote the number of partitions of $n$ with even (resp. odd) rank. It is well known that the difference is related to Ramanujan's mock theta function $f(q)$. This is the first example of a mock theta function that Ramanujan gave in his last letter to Hardy. Let

$$
\begin{aligned}
f(q) & =\sum_{n=0}^{\infty} a_{f}(n) q^{n}=1+\sum_{n=1}^{\infty} \frac{q^{n^{2}}}{(1+q)^{2}\left(1+q^{2}\right)^{2} \cdots\left(1+q^{n}\right)^{2}} \\
& =1+q-2 q^{2}+3 q^{3}-3 q^{4}+3 q^{5}-5 q^{6}+7 q^{7}-6 q^{8}+6 q^{9}-10 q^{10}+12 q^{11}-11 q^{12}+\cdots
\end{aligned}
$$

This function has been studied by many authors. Ramanujan conjectured an asymptotic formula for the coefficients $a_{f}(n)$. Dragonette [Dr52] improved this result by finding a Rademacher-type asymptotic expansion for the coefficients. The error term was subsequently improved by Andrews [An66], Bringmann and Ono [BrOn06], and Ahlgren and Dunn [AhDu19]. We have

$$
a_{f}(n)=N_{e}(n)-N_{o}(n),
$$

for $n \geq 0$.

Our main theorem is

Theorem 1.2. For all $\alpha \geq 3$ and all $n \geq 0$ we have

$$
a_{f}\left(5^{\alpha} n+\delta_{\alpha}\right)+a_{f}\left(5^{\alpha-2} n+\delta_{\alpha-2}\right) \equiv 0 \quad\left(\bmod 5^{\left\lfloor\frac{1}{2} \alpha\right\rfloor}\right),
$$

where $\delta_{\alpha}$ satisfies $0<\delta_{\alpha}<5^{\alpha}$ and $24 \delta_{\alpha} \equiv 1\left(\bmod 5^{\alpha}\right)$.

Since this paper was first written, we have found that Karl-Heinz Fricke [Fr13, p.9] independently observed (1.4) but without proof. Fricke [Fr13, p.232] also independently observed analogous congruences modulo powers of 7 and analogous congruences for Ramanujan's third order mock theta function

$$
\omega(q)=\sum_{n=0}^{\infty} \frac{q^{2 n(n+1)}}{(1+q)^{2}\left(1+q^{3}\right)^{2} \cdots\left(1+q^{2 n+1}\right)^{2}} .
$$

In a subsequent paper [CCG21] we will extend our methods to prove these other congruences.

Below in Section 3.A. we show that the generating function for

$$
a_{f}(5 n-1)+a_{f}(n / 5)
$$

is a linear combination of two eta-products. See Theorem 3.1. This enables us to use the theory of modular functions to obtain congruences. Our presentations and methods are similar to those of Paule and Radu [PaRa12], who solved a difficult conjecture of Sellers [Se94] for congruences modulo powers of 5 for Andrews's two-colored generalized Frobenius partitions [An84]. In Section 2. we include the necessary background and algorithms from the theory of modular functions for proving identities. In Section 3. we apply the theory of modular functions to prove our main theorem. In Section 4. we conclude the paper by discussing congruences modulo powers of 7 for both the rank and crank parity functions.

\section{Some Remarks and Notation}

Throughout this paper we use the standard $q$-notation. For finite products we use

$$
(z ; q)_{n}=(z)_{n}= \begin{cases}\prod_{j=0}^{n-1}\left(1-z q^{j}\right), & n>0 \\ 1, & n=0\end{cases}
$$


For infinite products we use

$$
\begin{gathered}
(z ; q)_{\infty}=(z)_{\infty}=\lim _{n \rightarrow \infty}(z ; q)_{n}=\prod_{n=1}^{\infty}\left(1-z q^{(n-1)}\right), \\
\left(z_{1}, z_{2}, \ldots, z_{k} ; q\right)_{\infty}=\left(z_{1} ; q\right)_{\infty}\left(z_{2} ; q\right)_{\infty} \cdots\left(z_{k} ; q\right)_{\infty}, \\
{[z ; q]_{\infty}=(z ; q)_{\infty}\left(z^{-1} q ; q\right)_{\infty}=\prod_{n=1}^{\infty}\left(1-z q^{(n-1)}\right)\left(1-z^{-1} q^{n}\right),} \\
{\left[z_{1}, z_{2}, \ldots, z_{k} ; q\right]_{\infty}=\left[z_{1} ; q\right]_{\infty}\left[z_{2} ; q\right]_{\infty} \cdots\left[z_{k} ; q\right]_{\infty},}
\end{gathered}
$$

for $|q|<1$ and $z, z_{1}, z_{2}, \ldots, z_{k} \neq 0$. For $\theta$-products we use

$$
J_{a, b}=\left(q^{a}, q^{b-a}, q^{b} ; q^{b}\right)_{\infty}, \quad \text { and } \quad J_{b}=\left(q^{b} ; q^{b}\right)_{\infty},
$$

and the Dedekind eta-function is given by

$$
\eta(\tau)=\exp (\pi i \tau / 12) \prod_{n=1}^{\infty}(1-\exp (2 \pi i n \tau))=q^{1 / 24} \prod_{n=1}^{\infty}\left(1-q^{n}\right)
$$

where $\operatorname{Im}(\tau)>0$.

Throughout this paper we let $\lfloor x\rfloor$ denote the largest integer less than or equal to $x$, and let $\lceil x\rceil$ denote the smallest integer greater than or equal to $x$.

We need some notation for formal Laurent series. See the remarks at the end of [PaRa12, Section 1, p.823]. Let $R$ be a ring and $q$ be an indeterminant. We let $R((q))$ denote the formal Laurent series in $q$ with coefficients in $R$. These are series of the form

$$
f=\sum_{n \in \mathbb{Z}} a_{n} q^{n}
$$

where $a_{n} \neq 0$ for at most finitely many $n<0$. For $f \neq 0$ we define the order of $f$ (with respect to $q$ ) as the smallest integer $N$ such that $a_{N} \neq 0$ and we write $N=\operatorname{ord}_{q}(f)$. We note that if $f$ is a modular function this coincides with $\operatorname{ord}(f, \infty)$. See equation (2.6) below for this other notation. Suppose $t$ and $f \in R((q))$ and the composition $f \circ t$ is well-defined as a formal Laurent series. This is the case if $\operatorname{ord}_{q}(t)>0$. The $t$-order of

$$
F=f \circ t=\sum_{n \in \mathbb{Z}} a_{n} t^{n}
$$

where $t=\sum_{n \in \mathbb{Z}} b_{n} q^{n}$, is defined to be the smallest integer $N$ such that $a_{N} \neq 0$ and we write $N=\operatorname{ord}_{t}(F)$. For example, if

$$
f=q^{-1}+1+2 q+\cdots, \quad t=q^{2}+3 q^{3}+5 q^{4}+\cdots,
$$

then

$$
F=f \circ t=t^{-1}+1+2 t+\cdots=q^{-2}-3 q^{-1}+5+\cdots,
$$

so that $\operatorname{ord}_{q}(f)=-1, \operatorname{ord}_{q}(t)=2, \operatorname{ord}_{t}(F)=-1$ and $\operatorname{ord}_{q}(F)=-2$.

\section{Modular Functions}

In this section we present the needed theory of modular functions which we use to prove identities.

A general reference is Rankin's book [Ra77]. 


\section{A. Background theory}

Our presentation is based on [Be91, pp.326-329]. Let $\mathscr{H}=\{\tau: \operatorname{Im}(\tau)>0\}$ (the complex upper halfplane). For each $M=\left(\begin{array}{ll}a & b \\ c & d\end{array}\right) \in M_{2}^{+}(\mathbb{Z})$, the set of integer $2 \times 2$ matrices with positive determinant, the bilinear transformation $M(\tau)$ is defined by

$$
M \tau=M(\tau)=\frac{a \tau+b}{c \tau+d} .
$$

The slash operator is defined by

$$
(f \mid M)(\tau)=f(M \tau)
$$

and satisfies

$$
f|M S=f| M \mid S,
$$

for matrices $M$ and $S$. The modular group $\Gamma(1)$ is defined by

$$
\Gamma(1)=\left\{\left(\begin{array}{ll}
a & b \\
c & d
\end{array}\right) \in M_{2}^{+}(\mathbb{Z}): a d-b c=1\right\} .
$$

We consider the following subgroups $\Gamma$ of the modular group with finite index

$$
\begin{gathered}
\Gamma_{0}(N)=\left\{\left(\begin{array}{ll}
a & b \\
c & d
\end{array}\right) \in \Gamma(1): c \equiv 0 \quad(\bmod N)\right\}, \\
\Gamma_{1}(N)=\left\{\left(\begin{array}{ll}
a & b \\
c & d
\end{array}\right) \in \Gamma(1):\left(\begin{array}{ll}
a & b \\
c & d
\end{array}\right) \equiv\left(\begin{array}{ll}
1 & * \\
0 & 1
\end{array}\right) \quad(\bmod N)\right\} .
\end{gathered}
$$

Such a group $\Gamma$ acts on $\mathscr{H} \cup \mathbb{Q} \cup \infty$ by the transformation $V(\tau)$, for $V \in \Gamma$ which induces an equivalence relation. We call a set $\mathscr{F} \subseteq \mathscr{H} \cup \mathbb{Q} \cup\{\infty\}$ a fundamental set for $\Gamma$ if it contains one element of each equivalence class. The finite set $\mathcal{F} \cap(\mathbb{Q} \cup\{\infty\})$ is called the complete set of inequivalent cusps.

A function $f: \mathscr{H} \longrightarrow \mathbb{C}$ is a modular function on $\Gamma$ if the following conditions hold:

(i) $f$ is holomorphic on $\mathscr{H}$.

(ii) $f \mid V=f$ for all $V \in \Gamma$.

(iii) For every $A \in \Gamma(1)$ the function $f \mid A^{-1}$ has an expansion

$$
\left(f \mid A^{-1}\right)(\tau)=\sum_{m=m_{0}}^{\infty} b(m) \exp (2 \pi i \tau m / \kappa)
$$

on some half-plane $\{\tau: \operatorname{Im} \tau>h \geq 0\}$, where $T=\left(\begin{array}{ll}1 & 1 \\ 0 & 1\end{array}\right)$ and

$$
\kappa=\min \left\{k>0: \pm A^{-1} T^{k} A \in \Gamma\right\} .
$$

The positive integer $\kappa=\kappa(\Gamma ; \zeta)$ is called the fan width of $\Gamma$ at the cusp $\zeta=A^{-1} \infty$. If $b\left(m_{0}\right) \neq 0$, then we write

$$
\operatorname{Ord}(f, \zeta, \Gamma)=m_{0}
$$

which is called the order of $f$ at $\zeta$ with respect to $\Gamma$. We also write

$$
\operatorname{ord}(f ; \zeta)=\frac{m_{0}}{\kappa}=\frac{m_{0}}{\kappa(\Gamma, \zeta)},
$$


which is called the invariant order of $f$ at $\zeta$. For each $z \in \mathscr{H}$, ord $(f ; z)$ denotes the order of $f$ at $z$ as an analytic function of $z$, and the order of $f$ with respect to $\Gamma$ is defined by

$$
\operatorname{Ord}(f, z, \Gamma)=\frac{1}{\ell} \operatorname{ord}(f ; z)
$$

where $\ell$ is the order of $z$ as a fixed point of $\Gamma$. We note $\ell=1,2$ or 3 . Our main tool for proving modular function identities is the valence formula [Ra77, Theorem 4.1.4, p.98]: If $f \neq 0$ is a modular function on $\Gamma$ and $\mathscr{F}$ is any fundamental set for $\Gamma$ then

$$
\sum_{z \in \mathscr{F}} \operatorname{Ord}(f, z, \Gamma)=0 .
$$

\section{B. Eta-product identities}

We will consider eta-products of the form

$$
f(\tau)=\prod_{d \mid N} \eta(d \tau)^{m_{d}}
$$

where $N$ is a positive integer, each $d>0$ and $m_{d} \in \mathbb{Z}$.

\section{Modularity}

Newman [Ne59] has found necessary and sufficient conditions under which an eta-product is a modular function on $\Gamma_{0}(N)$.

Theorem 2.1. ([Ne59, Theorem 4.7]) The function $f(\tau)$ (given in (2.8)) is a modular function on $\Gamma_{0}(N)$ if and only if

$$
\begin{aligned}
& \text { 1. } \sum_{d \mid N} m_{d}=0 \text {, } \\
& \text { 2. } \sum_{d \mid N} d m_{d} \equiv 0(\bmod 24) \text {, } \\
& \text { 3. } \sum_{d \mid N} \frac{N m_{d}}{d} \equiv 0(\bmod 24) \text {, and } \\
& \text { 4. } \prod_{d \mid N} d^{\left|m_{d}\right|} \text { is a square. }
\end{aligned}
$$

\section{Orders at cusps}

Ligozat [Li75] has computed the invariant order of an eta-product at the cusps of $\Gamma_{0}(N)$.

Theorem 2.2. ([Li75, Theorem 4.8]) If the eta-product $f(\tau)$ (given in (2.8)) is a modular function on $\Gamma_{0}(N)$, then its order at the cusp $\zeta=\frac{b}{c}$ (assuming $(b, c)=1$ ) is

$$
\operatorname{ord}(f(\tau) ; \zeta)=\sum_{d \mid N} \frac{(d, c)^{2} m_{d}}{24 d} .
$$

Chua and Lang [ChLa04] have found a set of inequivalent cusps for $\Gamma_{0}(N)$.

Theorem 2.3. ([ChLa04, p.354]) Let $N$ be a positive integer and for each positive divisor $d$ of $N$ let $e_{d}=(d, N / d)$. Then the set

$$
\Delta=\underset{d \mid N}{\cup} S_{d}
$$

is a complete set of inequivalent cusps of $\Gamma_{0}(N)$ where

$$
S_{d}=\left\{x_{i} / d:\left(x_{i}, d\right)=1, \quad 0 \leq x_{i} \leq d-1, \quad x_{i} \not \equiv x_{j} \quad\left(\bmod e_{d}\right)\right\} .
$$


Biagioli [Bi89] has found the fan width of the cusps of $\Gamma_{0}(N)$.

Lemma 2.4. ([Bi89, Lemma 4.2]) If $(r, s)=1$, then the fan width of $\Gamma_{0}(N)$ at $\frac{r}{s}$ is

$$
\kappa\left(\Gamma_{0}(N) ; \frac{r}{s}\right)=\frac{N}{\left(N, s^{2}\right)} .
$$

\section{An application of the valence formula}

Since eta-products have no zeros or poles in $\mathscr{H}$, the following result follows easily from the valence formula (2.7).

Theorem 2.5. Let $f_{1}(\tau), f_{2}(\tau), \ldots, f_{n}(\tau)$ be eta-products that are modular functions on $\Gamma_{0}(N)$. Let $\mathcal{S}_{N}$ be a set of inequivalent cusps for $\Gamma_{0}(N)$. Define the constant

$$
B=\sum_{\substack{\zeta \in \mathcal{S}_{N} \\ \zeta \neq \infty}} \min \left(\left\{\operatorname{Ord}\left(f_{j}, \zeta, \Gamma_{0}(N)\right): 1 \leq j \leq n\right\}\right),
$$

and consider

$$
g(\tau):=\alpha_{1} f_{1}(\tau)+\alpha_{2} f_{2}(\tau)+\cdots+\alpha_{n} f_{n}(\tau),
$$

where each $\alpha_{j} \in \mathbb{C}$. Then

$$
g(\tau) \equiv 0
$$

if and only if

$$
\operatorname{Ord}\left(g(\tau), \infty, \Gamma_{0}(N)\right)>-B .
$$

An algorithm for proving eta-product identities.

STEP 0. Write the identity in the following form:

$$
\alpha_{1} f_{1}(\tau)+\alpha_{2} f_{2}(\tau)+\cdots+\alpha_{n} f_{n}(\tau)=0,
$$

where each $\alpha_{i} \in \mathbb{C}$ and each $f_{i}(\tau)$ is an eta-product of level $N$.

STEP 1. Use Theorem 2.1 to check that $f_{j}(\tau)$ is a modular function on $\Gamma_{0}(N)$ for each $1 \leq j \leq n$. STEP 2. Use Theorem 2.3 to find a set $\mathcal{S}_{N}$ of inequivalent cusps for $\Gamma_{0}(N)$ and the fan width of each cusp.

STEP 3. Use Theorem 2.2 to calculate the order of each eta-product $f_{j}(\tau)$ at each cusp of $\Gamma_{0}(N)$.

STEP 4. Calculate

$$
B=\sum_{\substack{\zeta \in \mathcal{S}_{N} \\ \zeta \neq \infty}} \min \left(\left\{\operatorname{Ord}\left(f_{j}, \zeta, \Gamma_{0}(N)\right): 1 \leq j \leq n\right\}\right) .
$$

STEP 5. Show that

$$
\operatorname{Ord}\left(g(\tau), \infty, \Gamma_{0}(N)\right)>-B
$$

where

$$
g(\tau)=\alpha_{1} f_{1}(\tau)+\alpha_{2} f_{2}(\tau)+\cdots+\alpha_{n} f_{n}(\tau) .
$$

Theorem 2.5 then implies that $g(\tau) \equiv 0$ and hence the eta-product identity (2.13).

The third author has written a MAPLE package called ETA which implements this algorithm. See 


\section{A modular equation}

Define

$$
\begin{aligned}
t & :=t(\tau):=\frac{\eta(\tau)^{2} \eta(10 \tau)^{4}}{\eta(2 \tau)^{4} \eta(5 \tau)^{2}} \\
& =q-2 q^{2}+3 q^{3}-6 q^{4}+11 q^{5}-16 q^{6}+24 q^{7}-38 q^{8}+57 q^{9}-82 q^{10}+117 q^{11}+\cdots
\end{aligned}
$$

We note that $t(\tau)$ is a Hauptmodul for $\Gamma_{0}(10)$ [Ma09]. As an application of our algorithm we prove the following theorem which will be needed later.

Theorem 2.6. Let

$$
\begin{aligned}
& \sigma_{0}(\tau)=-t \\
& \sigma_{1}(\tau)=-5 t^{2}+2 \cdot 5 t \\
& \sigma_{2}(\tau)=-5^{2} t^{3}+2 \cdot 5^{2} t^{2}-7 \cdot 5 t \\
& \sigma_{3}(\tau)=-5^{3} t^{4}+2 \cdot 5^{3} t^{3}-7 \cdot 5^{2} t^{2}+12 \cdot 5 t \\
& \sigma_{4}(\tau)=-5^{4} t^{5}+2 \cdot 5^{4} t^{4}-7 \cdot 5^{3} t^{3}+12 \cdot 5^{2} t^{2}-11 \cdot 5 t
\end{aligned}
$$

where $t=t(\tau)$ is defined in (2.14). Then

$$
t(\tau)^{5}+\sum_{j=0}^{4} \sigma_{j}(5 \tau) t(\tau)^{j}=0 .
$$

Proof. From Theorem 2.1 we find that $t(\tau)$ is a modular function on $\Gamma_{0}(10)$ and $t(5 \tau)$ is a modular function on $\Gamma_{0}(50)$. Hence each term on the left side of (2.20) is a modular function on $\Gamma_{0}(50)$. For convenience we divide by $t(\tau)^{5}$ and let

$$
g(\tau)=1+\sum_{j=0}^{4} \sigma_{j}(5 \tau) t(\tau)^{j-5} .
$$

From Theorem 2.3, Lemma 2.4 and Theorem 2.2 we have the following table of fan widths for the cusps of $\Gamma_{0}(50)$, with the orders and invariant orders of both $t(\tau)$ and $t(5 \tau)$.

\begin{tabular}{|c|c|c|c|c|c|c|c|c|c|c|c|c|}
\hline$\zeta$ & 0 & $1 / 2$ & $1 / 5$ & $2 / 5$ & $3 / 5$ & $4 / 5$ & $1 / 10$ & $3 / 10$ & $7 / 10$ & $9 / 10$ & $1 / 25$ & $1 / 50$ \\
\hline$\kappa\left(\Gamma_{0}(50), \zeta\right)$ & 50 & 25 & 2 & 2 & 2 & 2 & 1 & 1 & 1 & 1 & 2 & 1 \\
\hline $\operatorname{ord}(t(\tau), \zeta)$ & 0 & $-1 / 5$ & 0 & 0 & 0 & 0 & 1 & 1 & 1 & 1 & 0 & 1 \\
\hline $\operatorname{Ord}\left(t(\tau), \zeta, \Gamma_{0}(50)\right)$ & 0 & -5 & 0 & 0 & 0 & 0 & 1 & 1 & 1 & 1 & 0 & 1 \\
\hline $\operatorname{ord}(t(5 \tau), \zeta)$ & 0 & $-1 / 25$ & 0 & 0 & 0 & 0 & -1 & -1 & -1 & -1 & 0 & 5 \\
\hline $\operatorname{Ord}\left(t(5 \tau), \zeta, \Gamma_{0}(50)\right)$ & 0 & -1 & 0 & 0 & 0 & 0 & -1 & -1 & -1 & -1 & 0 & 5 \\
\hline
\end{tabular}

Expanding the right side of (2.21) gives 16 terms of the form $t(5 \tau)^{k} t(\tau)^{j-5}$ with $1 \leq k \leq j+1$ where $0 \leq j \leq 4$, together with $(k, j)=(0,5)$. We calculate the order of each term at each cusp $\zeta$ of $\Gamma_{0}(50)$, and thus giving lower bounds for $\operatorname{Ord}\left(g(\tau), \zeta, \Gamma_{0}(50)\right.$ at each cusp in the following.

\begin{tabular}{|c|c|c|c|c|c|c|c|c|c|c|c|c|}
\hline$\zeta$ & 0 & $1 / 2$ & $1 / 5$ & $2 / 5$ & $3 / 5$ & $4 / 5$ & $1 / 10$ & $3 / 10$ & $7 / 10$ & $9 / 10$ & $1 / 25$ & $1 / 50$ \\
\hline $\operatorname{Ord}\left(g(\tau), \zeta, \Gamma_{0}(50)\right) \geq$ & 0 & 0 & 0 & 0 & 0 & 0 & -6 & -6 & -6 & -6 & 0 & 0 \\
\hline
\end{tabular}

Thus the constant $B$ in Theorem 2.5 is $B=-24$. It suffices to show that

$$
\operatorname{Ord}\left(g(\tau), \infty, \Gamma_{0}(50)\right)>24 .
$$

This is easily verified. Thus by Theorem 2.5 we have $g(\tau) \equiv 0$ and the result follows. 


\section{C. The $U_{p}$ operator}

Let $p$ be prime and

$$
f=\sum_{m=m_{0}}^{\infty} a(m) q^{m}
$$

be a formal Laurent series. We define $U_{p}$ by

$$
U_{p}(f):=\sum_{p m \geq m_{0}} a(p m) q^{m} .
$$

If $f$ is a modular function (with $q=\exp (2 \pi i \tau)$ ),

$$
U_{p}(f)=\frac{1}{p} \sum_{j=0}^{p} f \mid\left(\begin{array}{cc}
1 / p & j / p \\
0 & 1
\end{array}\right)=\frac{1}{p} \sum_{j=0}^{p} f\left(\frac{\tau+j}{p}\right) .
$$

By [AtLe70, Lemma 7, p.138] we have

Theorem 2.7. Let $p$ be prime. If $f$ is a modular function on $\Gamma_{0}(p N)$ and $p \mid N$, then $U_{p}(f)$ is a modular function on $\Gamma_{0}(N)$.

Gordon and Hughes [GoHu81, Theorem 4, p.336] have found lower bounds for the invariant orders of $U_{p}(f)$ at cusps. Let $\nu_{p}(n)$ denote the $p$-adic order of an integer $n$; i.e. the highest power of $p$ that divides $n$.

Theorem 2.8. ([GoHu81, Theorem 4]) Suppose $f(\tau)$ is a modular function on $\Gamma_{0}(p N)$, where $p$ is prime and $p \mid N$. Let $r=\frac{\beta}{\delta}$ be a cusp of $\Gamma_{0}(N)$, where $\delta \mid N$ and $(\beta, \delta)=1$. Then

$$
\operatorname{Ord}\left(U_{p}(f), r, \Gamma_{0}(N)\right) \geq \begin{cases}\frac{1}{p} \operatorname{Ord}\left(f, r / p, \Gamma_{0}(p N)\right) & \text { if } \nu_{p}(\delta) \geq \frac{1}{2} \nu_{p}(N) \\ \operatorname{Ord}\left(f, r / p, \Gamma_{0}(p N)\right) & \text { if } 0<\nu_{p}(\delta)<\frac{1}{2} \nu_{p}(N) \\ \min _{0 \leq k \leq p-1} \operatorname{Ord}\left(f,(r+k) / p, \Gamma_{0}(p N)\right) & \text { if } \nu_{p}(\delta)=0 .\end{cases}
$$

Theorems 2.5, 2.7 and 2.8 give the following algorithm.

\section{An algorithm for proving $U_{p}$ eta-product identities}

STEP $0 . \quad$ Write the identity in the form

$$
U_{p}\left(\alpha_{1} g_{1}(\tau)+\alpha_{2} g_{2}(\tau)+\cdots+\alpha_{k} g_{k}(\tau)\right)=\beta_{1} f_{1}(\tau)+\beta_{2} f_{2}(\tau)+\cdots+\beta_{n} f_{n}(\tau),
$$

where $p$ is prime, $p \mid N$, each $g_{j}(\tau)$ is an eta-product and a modular function on $\Gamma_{0}(p N)$, and each $f_{j}(\tau)$ is an eta-product and modular function on $\Gamma_{0}(N)$.

STEP 1. Use Theorem 2.1 to check that $f_{j}(\tau)$ is a modular function on $\Gamma_{0}(N)$ for each $1 \leq j \leq n$, and $g_{j}(\tau)$ is a modular function on $\Gamma_{0}(p N)$ for each $1 \leq j \leq k$.

STEP 2. Use Theorem 2.3 to find a set $\mathcal{S}_{N}$ of inequivalent cusps for $\Gamma_{0}(N)$ and the fan width of each cusp.

STEP 3a. Compute $\operatorname{Ord}\left(f_{j}, \zeta, \Gamma_{0}(N)\right)$ for each $j$ at each cusp $\zeta$ of $\Gamma_{0}(N)$ apart from $\infty$.

STEP 3b. Use Theorem 2.8 to find lower bounds $L\left(g_{j}, \zeta, N\right)$ for

$$
\operatorname{Ord}\left(U_{p}\left(g_{j}\right), \zeta, \Gamma_{0}(N)\right)
$$

for each cusp $\zeta$ of $\Gamma_{0}(N)$, and each $1 \leq j \leq k$. 
STEP 4. Calculate

$$
B=\sum_{\substack{\zeta \in \mathcal{S}_{N} \\ \zeta \neq \infty}} \min \left(\left\{\operatorname{Ord}\left(f_{j}, \zeta, \Gamma_{0}(N)\right): 1 \leq j \leq n\right\} \cup\left\{L\left(g_{j}, \zeta, N\right): 1 \leq j \leq k\right\}\right) .
$$

STEP 5. Show that

$$
\operatorname{Ord}\left(h(\tau), \infty, \Gamma_{0}(N)\right)>-B
$$

where

$$
h(\tau)=U_{p}\left(\alpha_{1} g_{1}(\tau)+\alpha_{2} g_{2}(\tau)+\cdots+\alpha_{k} g_{k}(\tau)\right)-\left(\beta_{1} f_{1}(\tau)+\beta_{2} f_{2}(\tau)+\cdots+\beta_{n} f_{n}(\tau)\right) .
$$

Theorem 2.5 then implies that $h(\tau) \equiv 0$ and hence the $U_{p}$ eta-product identity (2.24).

The third author has included an implementation of this algorithm in his ETA MAPLE package.

As an application of our algorithm we sketch the proof of

$$
U_{5}(g)=5 f_{1}(\tau)+2 f_{2}(\tau)
$$

where

$$
\begin{gathered}
g(\tau)=\frac{\eta(50 \tau)^{5} \eta(5 \tau)^{4} \eta(4 \tau)^{3} \eta(2 \tau)^{3}}{\eta(100 \tau)^{3} \eta(25 \tau)^{2} \eta(10 \tau)^{8} \eta(\tau)^{2}} \\
f_{1}(\tau)=\frac{\eta(10 \tau)^{8} \eta(\tau)^{4}}{\eta(5 \tau)^{4} \eta(2 \tau)^{8}}, \quad f_{2}(\tau)=\frac{\eta(10 \tau)^{5} \eta(\tau)^{2}}{\eta(20 \tau)^{3} \eta(5 \tau)^{2} \eta(4 \tau) \eta(2 \tau)} .
\end{gathered}
$$

We use Theorem 2.1 to check that $f_{j}(\tau)$ is a modular function on $\Gamma_{0}(20)$ for each $1 \leq j \leq 2$, and $g(\tau)$ is a modular function on $\Gamma_{0}(100)$. We use Theorem 2.3 to find a set $\mathcal{S}_{20}$ of inequivalent cusps for $\Gamma_{0}(20)$ and the fan width of each cusp. By Theorems 2.3, 2.2 and Lemma 2.4 we have the following table of orders.

\begin{tabular}{|c|c|c|c|c|c|c|}
\hline$\zeta$ & 0 & $1 / 2$ & $1 / 4$ & $1 / 5$ & $1 / 10$ & $1 / 20$ \\
\hline $\operatorname{Ord}\left(f_{1}(\tau), \zeta, \Gamma_{0}(20)\right)$ & 0 & -2 & -2 & 0 & 2 & 2 \\
\hline $\operatorname{Ord}\left(f_{2}(\tau), \zeta, \Gamma_{0}(20)\right)$ & 1 & 0 & -1 & 0 & 1 & -1 \\
\hline
\end{tabular}

Using Theorems 2.3,2.2, 2.8 and some calculation we have the following table of lower bounds $L(g, \zeta, 20)$.

\begin{tabular}{|c|c|c|c|c|c|c|}
\hline$\zeta$ & 0 & $1 / 2$ & $1 / 4$ & $1 / 5$ & $1 / 10$ & $1 / 20$ \\
\hline $\operatorname{Ord}\left(U_{5}(g), \zeta, \Gamma_{0}(20)\right) \geq$ & 0 & -2 & -2 & $-1 / 5$ & $3 / 5$ & $-6 / 5$ \\
\hline
\end{tabular}

Thus the constant $B$ in (2.25) is $B=-18 / 5$. It suffices to show that

$$
\operatorname{Ord}\left(h(\tau), \infty, \Gamma_{0}(20)\right) \geq 4,
$$

where

$$
h(\tau)=U_{5}(g)-\left(5 f_{1}(\tau)+2 f_{2}(\tau)\right) .
$$

This is easily verified. Thus by Theorem 2.5 we have $h(\tau) \equiv 0$ and the result (2.26) follows.

\section{D. Generalized eta-functions}

The generalized Dedekind eta function is defined to be

$$
\eta_{\delta, g}(\tau)=q^{\frac{\delta}{2} P_{2}(g / \delta)} \prod_{m \equiv \pm g}\left(1-q^{m}\right)
$$

where $P_{2}(t)=\{t\}^{2}-\{t\}+\frac{1}{6}$ is the second periodic Bernoulli polynomial, $\{t\}=t-[t]$ is the fractional part of $t, g, \delta, m \in \mathbb{Z}^{+}$and $0<g<\delta$. The function $\eta_{\delta, g}(\tau)$ is a modular function on $\mathrm{SL}_{2}(\mathbb{Z})$ with a 
multiplier system. Let $N$ be a fixed positive integer. A generalized Dedekind eta-product of level $N$ has the form

$$
f(\tau)=\prod_{\substack{\delta \mid N \\ 0<g<\delta}} \eta_{\delta, g}^{r \delta, g}(\tau)
$$

where

$$
r_{\delta, g} \in \begin{cases}\frac{1}{2} \mathbb{Z} & \text { if } g=\delta / 2, \\ \mathbb{Z} & \text { otherwise. }\end{cases}
$$

Robins [Ro94] has found sufficient conditions under which a generalized eta-product is a modular function on $\Gamma_{1}(N)$.

Theorem 2.9. ([Ro94, Theorem 3]) The function $f(\tau)$, defined in (2.28), is a modular function on $\Gamma_{1}(N)$ if

(i) $\sum_{\substack{\delta \mid N \\ g}} \delta P_{2}\left(\frac{g}{\delta}\right) r_{\delta, g} \equiv 0(\bmod 2)$, and

(ii) $\sum_{\substack{\delta \mid N \\ g}} \frac{N}{\delta} P_{2}(0) r_{\delta, g} \equiv 0(\bmod 2)$.

Cho, Koo and Park [CKP09] have found a set of inequivalent cusps for $\Gamma_{1}(N) \cap \Gamma_{0}(m N)$. The group $\Gamma_{1}(N)$ corresponds to the case $m=1$.

Theorem 2.10. ([CKP09, Corollary 4, p.930]) Let $a, c, a^{\prime}, c^{\prime} \in \mathbb{Z}$ with $(a, c)=\left(a^{\prime}, c^{\prime}\right)=1$.

(i) The cusps $\frac{a}{c}$ and $\frac{a^{\prime}}{c^{\prime}}$ are equivalent $\bmod \Gamma_{1}(N)$ if and only if

$$
\left(\begin{array}{l}
a^{\prime} \\
c^{\prime}
\end{array}\right) \equiv \pm\left(\begin{array}{c}
a+n c \\
c
\end{array}\right) \quad(\bmod N)
$$

for some integer $n$.

(ii) The following is a complete set of inequivalent cusps $\bmod \Gamma_{1}(N)$.

$$
\begin{aligned}
& \mathcal{S}=\left\{\frac{y_{c, j}}{x_{c, i}}: 0<c \mid N, 0<s_{c, i}, a_{c, j} \leq N,\left(s_{c, i}, N\right)=\left(a_{c, j}, N\right)=1,\right. \\
& s_{c, i}=s_{c, i^{\prime}} \Longleftrightarrow s_{c, 1} \equiv \pm s_{c^{\prime}, i^{\prime}} \quad\left(\bmod \frac{N}{c}\right), \\
& a_{c, j}=a_{c, j^{\prime}} \Longleftrightarrow \begin{cases}a_{c, j} \equiv \pm a_{c, j^{\prime}} \quad(\bmod c), & \text { if } c=\frac{N}{2} \text { or } N, \\
a_{c, j} \equiv a_{c, j^{\prime}} \quad(\bmod c), & \text { otherwise, }\end{cases} \\
& \left.x_{c, i}, y_{c, j} \in \mathbb{Z} \text { chosen so that } x_{c, i} \equiv c s_{c, i}, y_{c, j} \equiv a_{c, j} \quad(\bmod N),\left(x_{c, i}, y_{c, j}\right)=1\right\} \text {. }
\end{aligned}
$$

(iii) The fan width of the cusp $\frac{a}{c}$ is given by

$$
\kappa\left(\frac{a}{c}, \Gamma_{1}(N)\right)= \begin{cases}1, & \text { if } N=4 \text { and }(c, 4)=2, \\ \frac{N}{(c, N)}, & \text { otherwise. }\end{cases}
$$

In this theorem, it is understood as usual that the fraction $\frac{ \pm 1}{0}$ corresponds to $\infty$.

Robins [Ro94] has calculated the invariant order of $\eta_{\delta, g}(\tau)$ at any cusp. This gives a method for calculating the invariant order at any cusp of a generalized eta-product. 
Theorem 2.11. ([Ro94]) The order at the cusp $\zeta=\frac{a}{c}$ (assuming $(a, c)=1$ ) of the generalized eta-function $\eta_{\delta, g}(\tau)$ (defined in (2.27) and assuming $0<g<\delta$ ) is

$$
\operatorname{ord}\left(\eta_{\delta, g}(\tau) ; \zeta\right)=\frac{\varepsilon^{2}}{2 \delta} P_{2}\left(\frac{a g}{\varepsilon}\right)
$$

where $\varepsilon=(\delta, c)$.

\section{An algorithm for proving generalized eta-product identities}

We note that the analog of Theorem 2.5 holds for generalized eta-products which are modular functions on $\Gamma_{1}(N)$, and follows easily from the valence formula (2.7).

Theorem 2.12. ([FrGa19, Cor.2.5]) Let $f_{1}(\tau), f_{2}(\tau), \ldots, f_{n}(\tau)$ be generalized eta-products that are modular functions on $\Gamma_{1}(N)$. Let $\mathcal{S}_{N}$ be a set of inequivalent cusps for $\Gamma_{1}(N)$. Define the constant

$$
B=\sum_{\substack{s \in \mathcal{S}_{N} \\ s \neq \infty}} \min \left(\left\{\operatorname{Ord}\left(f_{j}, s, \Gamma_{1}(N)\right): 1 \leq j \leq n\right\} \cup\{0\}\right),
$$

and consider

$$
g(\tau):=\alpha_{1} f_{1}(\tau)+\alpha_{2} f_{2}(\tau)+\cdots+\alpha_{n} f_{n}(\tau)+1,
$$

where each $\alpha_{j} \in \mathbb{C}$. Then

$$
g(\tau) \equiv 0
$$

if and only if

$$
\operatorname{Ord}\left(g(\tau), \infty, \Gamma_{1}(N)\right)>-B
$$

The algorithm for proving generalized eta-product identities is completely analogous to the method for proving eta-product identities described in Section 2.B.. To prove an identity in the form

$$
\alpha_{1} f_{1}(\tau)+\alpha_{2} f_{2}(\tau)+\cdots+\alpha_{n} f_{n}(\tau)+1=0,
$$

the algorithm simply involves calculating the constant $B$ in (2.31) and then calculating enough coefficients to show that the inequality (2.33) holds. A more complete description is given in [FrGa19].

The third author has written a MAPLE package called thetaids which implements this algorithm. See

$$
\text { http://qseries.org/fgarvan/qmaple/thetaids/ }
$$

\section{The rank parity function modulo powers of 5}

\section{A. A Generating Function}

In this section we prove an identity for the generating function of

$$
a_{f}(5 n-1)+a_{f}(n / 5)
$$

where it is understood that $a_{f}(n)=0$ if $n$ is not a non-negative integer. Our proof depends on some results of Mao [Mao13] who found 5-dissection results for the rank modulo 10.

Theorem 3.1.

$$
\sum_{n=0}^{\infty}\left(a_{f}(5 n-1)+a_{f}(n / 5)\right) q^{n}=\frac{J_{2}^{4} J_{10}^{2}}{J_{1} J_{4}^{3} J_{20}}-4 q \frac{J_{1}^{2} J_{4}^{3} J_{5} J_{20}}{J_{2}^{5} J_{10}}
$$


Proof. From Watson [Wa36, p.64] we have

$$
f(q)=\frac{2}{(q ; q)_{\infty}} \sum_{n=-\infty}^{\infty} \frac{(-1)^{n} q^{n(3 n+1) / 2}}{1+q^{n}} .
$$

We find by replacing $n$ by $-n$ that

$$
\begin{aligned}
& \sum_{n=-\infty}^{\infty} \frac{(-1)^{n} q^{n(3 n+1) / 2+4 n}}{1+q^{5 n}}=\sum_{n=-\infty}^{\infty} \frac{(-1)^{n} q^{n(3 n+1) / 2}}{1+q^{5 n}}, \\
& \sum_{n=-\infty}^{\infty} \frac{(-1)^{n} q^{n(3 n+1) / 2+3 n}}{1+q^{5 n}}=\sum_{n=-\infty}^{\infty} \frac{(-1)^{n} q^{n(3 n+1) / 2+n}}{1+q^{5 n}} .
\end{aligned}
$$

By [Mao13, Lemma 3.1] we have

$$
\begin{aligned}
& \sum_{n=-\infty}^{\infty} \frac{(-1)^{n} q^{n(3 n+1) / 2}}{1+q^{5 n}}=P\left(q^{5},-q^{5} ; q^{25}\right)-\frac{P\left(q^{10},-q^{5} ; q^{25}\right)}{q^{3}}+\frac{(q ; q)_{\infty}}{J_{25}} \sum_{n=-\infty}^{\infty} \frac{(-1)^{n} q^{75 n(n+1) / 2+5}}{1+q^{25 n+5}}, \\
& \sum_{n=-\infty}^{\infty} \frac{(-1)^{n} q^{n(3 n+1) / 2+n}}{1+q^{5 n}}=P\left(q^{10},-q^{10} ; q^{25}\right)-q^{3} P\left(q^{5},-q^{10} ; q^{25}\right)-\frac{(q ; q)_{\infty}}{J_{25}} \sum_{n=-\infty}^{\infty} \frac{(-1)^{n} q^{75 n(n+1) / 2+8}}{1+q^{25 n+10}}, \\
& \sum_{n=-\infty}^{\infty} \frac{(-1)^{n} q^{n(3 n+1) / 2+2 n}}{1+q^{5 n}}=\frac{P\left(q^{5},-1 ; q^{25}\right)}{q^{6}}-\frac{P\left(q^{10},-1 ; q^{25}\right)}{q^{9}}-\frac{(q ; q)_{\infty}}{J_{25}} \sum_{n=-\infty}^{\infty} \frac{(-1)^{n} q^{25 n(3 n+1) / 2-1}}{1+q^{25 n}},
\end{aligned}
$$

where

$$
P(a, b ; q)=\frac{\left[a, a^{2} ; q\right]_{\infty}(q ; q)_{\infty}^{2}}{[b / a, a b, b ; q]_{\infty}} .
$$

From (3.35)-(3.39), and noting that $P\left(q^{5},-q^{5} ; q^{25}\right)=P\left(q^{10},-q^{10} ; q^{25}\right)$ we have

$$
\begin{aligned}
f(q)= & \frac{2}{(q ; q)_{\infty}} \sum_{n=-\infty}^{\infty} \frac{(-1)^{n} q^{n(3 n+1) / 2}}{1+q^{n}} \\
= & \frac{2}{(q ; q)_{\infty}} \sum_{n=-\infty}^{\infty} \frac{(-1)^{n} q^{n(3 n+1) / 2}\left(1-q^{n}+q^{2 n}-q^{3 n}+q^{4 n}\right)}{1+q^{5 n}} \\
= & \frac{2}{(q ; q)_{\infty}} \sum_{n=-\infty}^{\infty} \frac{(-1)^{n} q^{n(3 n+1) / 2}\left(2-2 q^{n}+q^{2 n}\right)}{1+q^{5 n}} \\
= & \frac{2}{J_{1}}\left\{2 q^{3} P\left(q^{5},-q^{10} ; q^{25}\right)-\frac{2 P\left(q^{10},-q^{5} ; q^{25}\right)}{q^{3}}+\frac{P\left(q^{5},-1 ; q^{25}\right)}{q^{6}}-\frac{P\left(q^{10},-1 ; q^{25}\right)}{q^{9}}\right\} \\
& +\frac{4}{J_{25}} \sum_{n=-\infty}^{\infty} \frac{(-1)^{n} q^{75 n(n+1) / 2+5}}{1+q^{25 n+5}}+\frac{4}{J_{25}} \sum_{n=-\infty}^{\infty} \frac{(-1)^{n} q^{75 n(n+1) / 2+8}}{1+q^{25 n+10}}-\frac{1}{q} f\left(q^{25}\right) .
\end{aligned}
$$

We let

$$
g(q)=\frac{2}{J_{1}}\left\{2 q^{3} P\left(q^{5},-q^{10} ; q^{25}\right)-\frac{2 P\left(q^{10},-q^{5} ; q^{25}\right)}{q^{3}}+\frac{P\left(q^{5},-1 ; q^{25}\right)}{q^{6}}-\frac{P\left(q^{10},-1 ; q^{25}\right)}{q^{9}}\right\},
$$

and write the 5-dissection of $g(q)$ as

$$
g(q)=g_{0}\left(q^{5}\right)+q g_{1}\left(q^{5}\right)+q^{2} g_{2}\left(q^{5}\right)+q^{3} g_{3}\left(q^{5}\right)+q^{4} g_{4}\left(q^{5}\right) .
$$


From (3.35), (3.42) and (3.43), replacing $q^{5}$ by $q$, we have

$$
\sum_{n=0}^{\infty} a_{f}(5 n+4) q^{n}=-\frac{1}{q} f\left(q^{5}\right)+g_{4}(q),
$$

after dividing both sides by $q^{4}$ and replacing $q^{5}$ by $q$.

The 5 -dissection of $J_{1}$ is well-known

$$
J_{1}=J_{25}\left(B\left(q^{5}\right)-q-q^{2} \frac{1}{B\left(q^{5}\right)}\right),
$$

where

$$
B(q)=\frac{J_{2,5}}{J_{1,5}} .
$$

See for example [Ga88, Lemma (3.18)].

From (3.42), (3.43) and (3.45)

$$
\begin{aligned}
& J_{25}\left(g_{0}\left(q^{5}\right)+q g_{1}\left(q^{5}\right)+q^{2} g_{2}\left(q^{5}\right)+q^{3} g_{3}\left(q^{5}\right)+q^{4} g_{4}\left(q^{5}\right)\right)\left(B\left(q^{5}\right)-q-q^{2} \frac{1}{B\left(q^{5}\right)}\right) \\
& =4 q^{3} P\left(q^{5},-q^{10} ; q^{25}\right)-\frac{4 P\left(q^{10},-q^{5} ; q^{25}\right)}{q^{3}}+\frac{2 P\left(q^{5},-1 ; q^{25}\right)}{q^{6}}-\frac{2 P\left(q^{10},-1 ; q^{25}\right)}{q^{9}} .
\end{aligned}
$$

By expanding the left side of (3.46) and comparing both sides according to the residue of the exponent of $q$ modulo 5 , we obtain 5 equations:

$$
\begin{aligned}
& B(q) g_{0}-q g_{4}-\frac{q}{B(q)} g_{3}=0 \\
& B(q) g_{1}-g_{0}-\frac{q}{B(q)} g_{4}=-\frac{2 P\left(q^{2},-1 ; q^{5}\right)}{q^{2} J_{5}}, \\
& B(q) g_{2}-g_{1}-\frac{1}{B(q)} g_{0}=-\frac{4 P\left(q^{2},-q ; q^{5}\right)}{q J_{5}}, \\
& B(q) g_{3}-g_{2}-\frac{1}{B(q)} g_{1}=\frac{4 P\left(q,-q^{2} ; q^{5}\right)}{J_{5}} \\
& B(q) g_{4}-g_{3}-\frac{1}{B(q)} g_{2}=\frac{2 P\left(q,-1 ; q^{5}\right)}{q^{2} J_{5}}
\end{aligned}
$$

where $g_{j}=g_{j}(q)$ for $0 \leq j \leq 4$.

Solving these equations we find that

$$
\begin{aligned}
g_{4}(q) & =\frac{1}{J_{5}\left(B^{5}-11 q-q^{2} / B^{5}\right)}\left(\frac{2}{q^{2}} X_{2} B^{4}-\frac{2}{q} X_{1} B^{-4}+4 X_{4} B^{3}+4 X_{3} B^{-3}\right. \\
& \left.-\frac{8}{q} X_{3} B^{2}+8 q X_{4} B^{-2}-\frac{6}{q^{2}} X_{1} B-\frac{6}{q} X_{2} B^{-1}\right),
\end{aligned}
$$

where $B:=B(q)$ and

$$
\begin{array}{ll}
X_{1}=P\left(q^{2},-1 ; q^{5}\right)=\frac{q^{2} J_{1,10} J_{2,10}^{3} J_{3,10}^{3} J_{5,10}^{2}}{2 J_{10}^{6} J_{4,10}}, & X_{2}=P\left(q,-1 ; q^{5}\right)=\frac{q J_{1,10}^{3} J_{3,10} J_{4,10}^{3} J_{5,10}^{2}}{2 J_{10}^{6} J_{2,10}}, \\
X_{3}=P\left(q^{2},-q ; q^{5}\right)=\frac{q J_{1,10}^{3} J_{3,10}^{2} J_{4,10}^{2} J_{5,10}}{J_{10}^{6}}, & X_{4}=P\left(q,-q^{2} ; q^{5}\right)=\frac{J_{1,10}^{2} J_{2,10}^{2} J_{3,10}^{3} J_{5,10}}{J_{10}^{6}} .
\end{array}
$$


The following identity is also well-known

$$
\frac{J_{1}^{6}}{J_{5}^{6}}=B^{5}-11 q-q^{2} \frac{1}{B^{5}} .
$$

See for example [HiHu81, Lemma (2.5)].

By (3.52) and (3.53), we have

$$
\begin{aligned}
g_{4}(q)= & \frac{J_{1,10}^{3} J_{3,10} J_{4,10}^{3} J_{5,10}^{2} J_{2,5}^{4} J_{5}^{5}}{q J_{2,10} J_{1,5}^{4} J_{10}^{6} J_{1}^{6}}-\frac{q J_{1,10} J_{2,10}^{3} J_{3,10}^{3} J_{5,10}^{2} J_{1,5}^{4} J_{5}^{5}}{J_{4,10} J_{2,5}^{4} J_{10}^{6} J_{1}^{6}}+4 \frac{J_{1,10}^{2} J_{2,10}^{2} J_{3,10}^{3} J_{5,10} J_{2,5}^{3} J_{5}^{5}}{J_{1,5}^{3} J_{10}^{6} J_{1}^{6}} \\
& +4 \frac{q J_{1,10}^{3} J_{3,10}^{2} J_{4,10}^{2} J_{5,10} J_{1,5}^{3} J_{5}^{5}}{J_{2,5}^{3} J_{10}^{6} J_{1}^{6}}-8 \frac{J_{1,10}^{3} J_{3,10}^{2} J_{4,10}^{2} J_{5,10} J_{2,5}^{2} J_{5}^{5}}{J_{1,5}^{2} J_{10}^{6} J_{1}^{6}}+8 \frac{q J_{1,10}^{2} J_{2,10}^{2} J_{3,10}^{3} J_{5,10} J_{1,5}^{2} J_{5}^{5}}{J_{2,5}^{2} J_{10}^{6} J_{1}^{6}} \\
& -3 \frac{J_{1,10} J_{2,10}^{3} J_{3,10}^{3} J_{5,10}^{2} J_{2,5} J_{5}^{5}}{J_{4,10} J_{1,5} J_{10}^{6} J_{1}^{6}}-3 \frac{J_{1,10}^{3} J_{3,10} J_{4,10}^{3} J_{5,10}^{2} J_{1,5} J_{5}^{5}}{J_{2,10} J_{2,5} J_{10}^{6} J_{1}^{6}}
\end{aligned}
$$

We prove

$$
g_{4}(q)=-4 \frac{J_{1}^{2} J_{4}^{3} J_{5} J_{20}}{J_{2}^{5} J_{10}}+\frac{1}{q} \frac{J_{2}^{4} J_{10}^{2}}{J_{1} J_{4}^{3} J_{20}}
$$

using the algorithm described in Section 2.D. We first use (3.54) to rewrite (3.55) as the following modular function identity for generalized eta-products on $\Gamma_{1}(20)$.

$$
\begin{aligned}
0= & -\frac{\eta_{10,1}^{6} \eta_{10,4}^{4}}{\eta_{10,2}^{4} \eta_{10,3}^{6}}+4 \frac{\eta_{10,2}^{2} \eta_{10,3}}{\eta_{10,4}^{2} \eta_{10,5}}+4 \frac{\eta_{10,1}^{7} \eta_{10,4}^{6}}{\eta_{10,2}^{6} \eta_{10,3}^{6} \eta_{10,5}}-8 \frac{\eta_{10,1}^{2} \eta_{10,4}}{\eta_{10,2} \eta_{10,3} \eta_{10,5}}+8 \frac{\eta_{10,1}^{5} \eta_{10,4}^{3}}{\eta_{10,2}^{3} \eta_{10,3}^{4} \eta_{10,5}}-3 \frac{\eta_{10,1} \eta_{10,2}}{\eta_{10,3} \eta_{10,4}} \\
& -3 \frac{\eta_{5,1}^{5}}{\eta_{5,2}^{5}}+4 \frac{\eta_{20,1}^{9} \eta_{20,3}^{3} \eta_{20,4}^{7} \eta_{20,6}^{4} \eta_{20,7}^{3} \eta_{20,8}^{3} \eta_{20,9}^{9}}{\eta_{20,10}^{2}}-\frac{\eta_{20,1}^{6} \eta_{20,2}^{6} \eta_{20,4}^{7} \eta_{20,6}^{10} \eta_{20,8}^{3} \eta_{20,9}^{6} \eta_{20,10}^{2}}{\eta_{20,5}^{4}}
\end{aligned}
$$

We use Theorem 2.9 to check that each generalized eta-product is a modular function on $\Gamma_{1}(20)$. We then use Theorems 2.10 and 2.11 to calculate the order of each generalized eta-product at each cusp of $\Gamma_{1}(20)$. We calculate the constant in equation (2.31) to find that $B=24$. We let $g(\tau)$ be the right side of (3.56) and easily show that $\operatorname{Ord}\left(g(\tau), \infty, \Gamma_{1}(20)\right)>24$. The required identity follows by Theorem 2.12 .

From (3.44) and (3.55) we have

$$
\sum_{n=0}^{\infty} a_{f}(5 n-1) q^{n}+f\left(q^{5}\right)=q g_{4}(q)=\frac{J_{2}^{4} J_{10}^{2}}{J_{1} J_{4}^{3} J_{20}}-4 q \frac{J_{1}^{2} J_{4}^{3} J_{5} J_{20}}{J_{2}^{5} J_{10}}
$$

which is our result (3.34).

\section{B. A Fundamental Lemma}

We need the following fundamental lemma, whose proof follows easily from Theorem 2.6.

Lemma 3.2. (A Fundamental Lemma) Suppose $u=u(\tau)$, and $j$ is any integer. Then

$$
U_{5}\left(u t^{j}\right)=-\sum_{l=0}^{4} \sigma_{l}(\tau) U_{5}\left(u t^{j+l-5}\right),
$$

where $t=t(\tau)$ is defined in (2.14) and the $\sigma_{j}(\tau)$ are given in (2.15)-(2.19).

Proof. The result follows easily from (2.20) by multiplying both sides by $u t^{j-5}$ and applying $U_{5}$. 
Lemma 3.3. Let $u=u(\tau)$, and $l \in \mathbb{Z}$. Suppose for $l \leq k \leq l+4$ there exist Laurent polynomials $p_{u, k}(t) \in \mathbb{Z}\left[t, t^{-1}\right]$ such that

$$
U_{5}\left(u t^{k}\right)=v p_{u, k}(t)
$$

and

$$
\operatorname{ord}_{t}\left(p_{u, k}(t)\right) \geq\left\lceil\frac{k+s}{5}\right\rceil
$$

for a fixed integer $s$, where $t=t(\tau)$ is defined in (2.14) and where $v=v(\tau)$. Then there exists a sequence of Laurent polynomials $p_{u, k}(t) \in \mathbb{Z}\left[t, t^{-1}\right], k \in \mathbb{Z}$, such that (3.58) and (3.59) hold for all $k \in \mathbb{Z}$.

Proof. We proceed by induction on $k$. Let $N>l+4$ and assume the result holds for $l \leq k \leq N-1$. Then by Lemma 3.2 we have

$$
U_{5}\left(u t^{N}\right)=-\sum_{j=0}^{4} \sigma_{j}(\tau) U_{5}\left(u t^{N+j-5}\right)=-v \sum_{j=0}^{4} \sigma_{j}(\tau) p_{u, N+j-5}(t)=v p_{u, N}(t),
$$

where

$$
p_{u, N}(t)=-\sum_{j=0}^{4} \sigma_{j}(\tau) p_{u, N+j-5}(t) \in \mathbb{Z}\left[t, t^{-1}\right]
$$

and

$$
\begin{aligned}
\operatorname{ord}_{t}\left(p_{u, N}(t)\right) & \geq \min _{0 \leq j \leq 4}\left(\operatorname{ord}_{t}\left(\sigma_{j}\right)+\operatorname{ord}_{t}\left(p_{u, N+j-5}(t)\right)\right) \\
& \geq \min _{0 \leq j \leq 4}\left(1+\left\lceil\frac{N+j+s-5}{5}\right\rceil\right)=\left\lceil\frac{N+s}{5}\right\rceil .
\end{aligned}
$$

The result for all $k \geq l$ follows. The induction proof for $k<l$ is similar.

Lemma 3.4. Let $u=u(\tau)$, and $l \in \mathbb{Z}$. Suppose for $l \leq k \leq l+4$ there exist Laurent polynomials $p_{u, k}(t) \in \mathbb{Z}\left[t, t^{-1}\right]$ such that

$$
U_{5}\left(u t^{k}\right)=v p_{u, k}(t)
$$

where

$$
p_{u, k}(t)=\sum_{n} c_{u}(k, n) t^{n}, \quad \nu_{5}\left(c_{u}(k, n)\right) \geq\left\lfloor\frac{3 n-k+r}{4}\right\rfloor
$$

for a fixed integer $r$, where $t=t(\tau)$ is defined in (2.14) and where $v=v(\tau)$. Then there exists a sequence of Laurent polynomials $p_{u, k}(t) \in \mathbb{Z}\left[t, t^{-1}\right], k \in \mathbb{Z}$, such that (3.60) holds for $k>l+4$, where

$$
p_{u, k}(t)=\sum_{n} c_{u}(k, n) t^{n}, \quad \text { and } \quad \nu_{5}\left(c_{u}(k, n)\right) \geq\left\lfloor\frac{3 n-k+r+2}{4}\right\rfloor .
$$

Remark 3.5. Recall that $\nu_{p}(n)$ denotes the $p$-adic order of an integer $n$; i.e. the highest power of $p$ that divides $n$.

Proof. We proceed by induction on $k$. Let $N>l+4$ and assume (3.60) holds for $l \leq k \leq N-1$ where

$$
p_{u, k}(t)=\sum_{n} c_{u}(k, n) t^{n}, \quad \nu_{5}\left(c_{u}(k, n)\right)=\left\lfloor\frac{3 n-k+r}{4}\right\rfloor .
$$


As in the proof of Lemma 3.3 we have

$$
U_{5}\left(u t^{N}\right)=v p_{u, N}(t)
$$

where

$$
p_{u, N}(t)=-\sum_{j=0}^{4} \sigma_{j}(\tau) p_{u, N+j-5}(t) \in \mathbb{Z}\left[t, t^{-1}\right],
$$

From Lemma 3.2 we have

$$
\sigma_{j}(\tau)=\sum_{l=1}^{j+1} s(j, l) t^{l} \in \mathbb{Z}[t]
$$

where

$$
\nu_{5}(s(j, l)) \geq\left\lfloor\frac{3 l+j}{4}\right\rfloor
$$

for $1 \leq l \leq j+1,0 \leq j \leq 4$. Therefore

$$
p_{u, N}(t)=-\sum_{j=0}^{4} \sum_{l=1}^{j+1} s(j, l) \sum_{m} c_{u}(N+j-5, m) t^{m+l}=\sum_{n} c_{u}(N, n) t^{n},
$$

where

$$
c_{u}(N, n)=-\sum_{j=0}^{4} \sum_{l=1}^{j+1} s(j, l) c_{u}(N+j-5, n-l),
$$

and

$$
\begin{aligned}
\nu_{5}\left(c_{u}(N, n)\right) & \geq \min _{\substack{1 \leq l \leq j+1 \\
0 \leq j \leq 4}}\left(\nu_{5}(s(j, l))+\nu_{5}\left(c_{u}(N+j-5, n-l)\right)\right. \\
& \geq \min _{\substack{1 \leq l \leq j+1 \\
0 \leq j \leq 4}}\left(\left\lfloor\frac{3 l+j}{4}\right\rfloor+\left\lfloor\frac{3(n-l)-(N+j-5)+r}{4}\right\rfloor\right) \\
& \geq \min _{\substack{1 \leq l \leq j+1 \\
0 \leq j \leq 4}}\left\lfloor\frac{3 l+j+3(n-l)-(N+j-5)+r-3}{4}\right\rfloor \geq\left\lfloor\frac{3 n-N+r+2}{4}\right\rfloor .
\end{aligned}
$$

The result follows.

We define the following functions which will be needed in the proof of Theorem 1.2.

$$
P_{A}:=\frac{J_{10}^{2} J_{5} J_{2}^{6}}{J_{20} J_{4}^{3} J_{1}^{5}}-4 \frac{q J_{20} J_{5}^{2} J_{4}^{3}}{J_{10} J_{2}^{3} J_{1}^{2}}, \quad P_{B}:=\frac{J_{10}^{6} J_{2}^{2} J_{1}}{q J_{20}^{3} J_{5}^{5} J_{4}}+4 \frac{q J_{20}^{3} J_{4} J_{1}^{2}}{J_{10}^{3} J_{5}^{2} J_{2}} \quad A:=\frac{J_{50}^{2} J_{1}^{4}}{J_{25}^{4} J_{2}^{2}}, \quad B:=\frac{q J_{25}}{J_{1}} .
$$

For $f=f(\tau)$ we define

$$
U_{A}(f):=U_{5}(A f), \quad U_{B}(f):=U_{5}(B f) .
$$

First we need some initial values of $U_{A}\left(P_{A} t^{k}\right)$ and $U_{B}\left(P_{B} t^{k}\right)$.

\section{Lemma 3.6.}

\section{Group I}

$$
\begin{aligned}
& U_{A}\left(P_{A}\right)=P_{B}\left(5^{4} t^{5}-7 \cdot 5^{3} t^{4}+14 \cdot 5^{2} t^{3}-2 \cdot 5^{2} t^{2}+t\right), \\
& U_{A}\left(P_{A} t^{-1}\right)=-P_{B} t \\
& U_{A}\left(P_{A} t^{-2}\right)=-5 P_{B} t^{2}
\end{aligned}
$$




$$
\begin{aligned}
& U_{A}\left(P_{A} t^{-3}\right)=-5^{2} P_{B} t^{3}, \\
& U_{A}\left(P_{A} t^{-4}\right)=-5^{3} P_{B} t^{4} .
\end{aligned}
$$

\section{Group II}

$$
\begin{aligned}
& U_{B}\left(P_{B}\right)=P_{A}, \\
& U_{B}\left(P_{B} t^{-1}\right)=P_{A}(-5 t+2), \\
& U_{B}\left(P_{B} t^{-2}\right)=P_{A}\left(5^{2} t^{2}-8 \cdot 5 t+8\right), \\
& U_{B}\left(P_{B} t^{-3}\right)=P_{A}\left(5^{3} t^{3}-34 \cdot 5 t+34\right), \\
& U_{B}\left(P_{B} t^{-4}\right)=P_{A}\left(-5^{4} t^{4}+16 \cdot 5^{3} t^{3}-36 \cdot 5^{2} t^{2}-128 \cdot 5 t+6 \cdot 5^{2}\right) .
\end{aligned}
$$

Proof. We use the algorithm described in Section 2.C. to prove each of these identities. The identities take the form

$$
U_{5}(g)=f,
$$

where $f, g$ are linear combinations of eta-products. For each identity we check that $f$ is a linear combination of eta-products which are modular functions on $\Gamma_{0}(20)$ and that $g$ is a linear combination of eta-products which are modular functions on $\Gamma_{0}(100)$. For each of the identities we follow the 5 steps in the algorithm given after Theorem 2.8. We note that the smallest value of the constant $B$ (defined in equation (2.25)) encountered is $B=-14$. These steps have been carried out with the help of MAPLE, including all necessary verifications so that the results are proved.

Following [PaRa12] a map $a: \mathbb{Z} \times \mathbb{Z} \longrightarrow \mathbb{Z}$ is called a discrete array if for each $i$ the map $a(i,-): \mathbb{Z} \longrightarrow \mathbb{Z}$, by $j \mapsto a(i, j)$ has finite support.

Lemma 3.7. There exist discrete arrays $a$ and $b$ such that for $k \geq 1$

$$
\begin{aligned}
& U_{A}\left(P_{A} t^{k}\right)=P_{B} \sum_{n \geq\lceil(k+5) / 5\rceil} a(k, n) t^{n}, \quad \text { where } \quad \nu_{5}(a(k, n)) \geq\left\lfloor\frac{3 n-k}{4}\right\rfloor, \\
& U_{B}\left(P_{B} t^{k}\right)=P_{A} \sum_{n \geq\lceil k / 5\rceil} b(k, n) t^{n}, \quad \text { where } \quad \nu_{5}(b(k, n)) \geq\left\lfloor\frac{3 n-k+2}{4}\right\rfloor .
\end{aligned}
$$

Proof. From Lemma 3.6, Group I we find there is a discrete array a such that

$$
U_{A}\left(P_{A} t^{k}\right)=P_{B} \sum_{n \geq\lceil(k+5) / 5\rceil} a(k, n) t^{n}, \quad \text { where } \quad \nu_{5}(a(k, n)) \geq\left\lfloor\frac{3 n-k-2}{4}\right\rfloor,
$$

for $-4 \leq k \leq 0$. Lemma 3.3 (with $s=4$ ) and Lemma 3.4 (with $r=-2$ ) imply (3.63) for $k \geq 1$. From Lemma 3.6, Group II we find there is a discrete array $b$ such that

$$
U_{B}\left(P_{B} t^{k}\right)=P_{A} \sum_{n \geq\lceil k / 5\rceil} b(k, n) t^{n}, \quad \text { where } \quad \nu_{5}(b(k, n)) \geq\left\lfloor\frac{3 n-k}{4}\right\rfloor,
$$

for $-4 \leq k \leq 0$. Lemma 3.3 (with $s=0$ ) and Lemma 3.4 (with $r=0$ ) imply (3.64) for $k \geq 1$.

\section{C. Proof of Theorem 1.2}

For $\alpha \geq 1$ define an integer $\delta_{\alpha}$ by $0<\delta_{\alpha}<5^{\alpha}$ and $24 \delta_{\alpha} \equiv 1\left(\bmod 5^{\alpha}\right)$. Then

$$
\delta_{2 \alpha}=\frac{23 \times 5^{2 \alpha}+1}{24}, \quad \delta_{2 \alpha+1}=\frac{19 \times 5^{2 \alpha+1}+1}{24} .
$$


We let

$$
\lambda_{2 \alpha}=\lambda_{2 \alpha+1}=\frac{5}{24}\left(1-5^{2 \alpha}\right) .
$$

For $n \geq 0$ we define

$$
c_{f}(n):=a_{f}(5 n-1)+a_{f}(n / 5) .
$$

We find that for $\alpha \geq 3$

$$
\sum_{n=0}^{\infty}\left(a_{f}\left(5^{\alpha} n+\delta_{\alpha}\right)+a_{f}\left(5^{\alpha-2} n+\delta_{\alpha-2}\right)\right) q^{n+1}=\sum_{n=1}^{\infty} c_{f}\left(5^{\alpha-1} n+\lambda_{\alpha-1}\right) q^{n} .
$$

We define the sequence of functions $\left(L_{\alpha}\right)_{\alpha=0}^{\infty}$ by $L_{0}:=P_{A}$ and for $\alpha \geq 0$

$$
L_{2 \alpha+1}:=U_{A}\left(L_{2 \alpha}\right), \quad \text { and } \quad L_{2 \alpha+2}:=U_{B}\left(L_{2 \alpha+1}\right) .
$$

Lemma 3.8. For $\alpha \geq 0$,

$$
L_{2 \alpha}=\frac{J_{5} J_{2}^{2}}{J_{1}^{4}} \sum_{n=0}^{\infty} c_{f}\left(5^{2 \alpha} n+\lambda_{2 \alpha}\right) q^{n}
$$

and

$$
L_{2 \alpha+1}=\frac{J_{10}^{2} J_{1}}{J_{5}^{4}} \sum_{n=0}^{\infty} c_{f}\left(5^{2 \alpha+1} n+\lambda_{2 \alpha+1}\right) q^{n} .
$$

Proof. By Theorem 3.1 we have

$$
\begin{aligned}
L_{0} & =P_{A}=\frac{J_{10}^{2} J_{5} J_{2}^{6}}{J_{20} J_{4}^{3} J_{1}^{5}}-4 q \frac{J_{20} J_{5}^{2} J_{4}^{3}}{J_{10} J_{2}^{3} J_{1}^{2}}=\frac{J_{5} J_{2}^{2}}{J_{1}^{4}}\left(\frac{J_{10}^{2} J_{2}^{4}}{J_{20} J_{4}^{3} J_{1}}-4 q \frac{J_{20} J_{5} J_{4}^{3} J_{1}^{2}}{J_{10} J_{2}^{5}}\right) \\
& =\frac{J_{5} J_{2}^{2}}{J_{1}^{4}} \sum_{n=0}^{\infty}\left(a_{f}(5 n-1)+a_{f}(n / 5)\right) q^{n}=\frac{J_{5} J_{2}^{2}}{J_{1}^{4}} \sum_{n=0}^{\infty} c_{f}\left(n+\lambda_{0}\right) q^{n},
\end{aligned}
$$

since $\lambda_{0}=0$.

$$
\begin{aligned}
L_{1} & =U_{A}\left(L_{0}\right)=U_{5}\left(A L_{0}\right)=U_{5}\left(\frac{J_{50}^{2} J_{5}}{J_{25}^{4}} \sum_{n=0}^{\infty} c_{f}(n) q^{n}\right) \\
& =\frac{J_{10}^{2} J_{1}}{J_{5}^{4}} \sum_{n=0}^{\infty} c_{f}\left(5 n+\lambda_{1}\right) q^{n},
\end{aligned}
$$

since $\lambda_{1}=0$. This is the second equation with $\alpha=0$. The general result follows similarly using a routine induction argument, by noting that

$$
5 \lambda_{\alpha-1}-1=\delta_{\alpha}-5^{\alpha}, \quad \frac{1}{5} \lambda_{\alpha-1}=\delta_{\alpha-2} .
$$

Our main result for the rank parity function modulo powers of 5 is the following theorem.

Theorem 3.9. There exists a discrete array $\ell$ such that for $\alpha \geq 1$

$$
\begin{aligned}
L_{2 \alpha} & =P_{A} \sum_{n \geq 1} \ell(2 \alpha, n) t^{n}, \quad \text { where } \quad \nu_{5}(\ell(2 \alpha, n)) \geq \alpha+\left\lfloor\frac{3 n-3}{4}\right\rfloor, \\
L_{2 \alpha+1} & =P_{B} \sum_{n \geq 2} \ell(2 \alpha+1, n) t^{n}, \quad \text { where } \quad \nu_{5}(\ell(2 \alpha+1, n)) \geq \alpha+1+\left\lfloor\frac{3 n-6}{4}\right\rfloor .
\end{aligned}
$$


Proof. We define the discrete array $\ell$ recursively. Define

$$
\begin{aligned}
& \ell(1,1)=1, \ell(1,2)=-2 \cdot 5^{2}, \ell(1,3)=14 \cdot 5^{2}, \ell(1,4)=-7 \cdot 5^{3}, \ell(1,5)=5^{4}, \\
& \text { and } \ell(1, k)=0, \quad \text { for } k \geq 6 .
\end{aligned}
$$

For $\alpha \geq 1$ define

$$
\ell(2 \alpha, n)=\sum_{k \geq 1} \ell(2 \alpha-1, k) b(k, n) \quad(\text { for } n \geq 1)
$$

and

$$
\ell(2 \alpha+1, n)=\sum_{k \geq 1} \ell(2 \alpha, k) a(k, n) \quad(\text { for } n \geq 2),
$$

where $a$ and $b$ are the discrete arrays given in Lemma 3.7. From Lemma 3.6, Group I and by Lemma 3.7 and equation (3.69) we have

$$
\begin{aligned}
L_{1}=U_{A}\left(L_{0}\right)=U_{A}\left(P_{A}\right) & =P_{B} \sum_{n=1}^{5} \ell(1, n) t^{n}, \quad \text { where } \quad \nu_{5}(\ell(1, n)) \geq\left\lfloor\frac{3 n-2}{4}\right\rfloor . \\
L_{2} & =U_{B}\left(L_{1}\right)=\sum_{n=1}^{5} \ell(1, n) U_{B}\left(P_{B} t^{n}\right), \\
& =\sum_{n=1}^{5} \ell(1, n) P_{A} \sum_{k \geq 1} b(n, k) t^{k} \\
& =P_{A} \sum_{n \geq 1} \sum_{k=1}^{5} \ell(1, k) b(k, n) t^{n} \\
& =P_{A} \sum_{n \geq 1} \ell(2, n) t^{n},
\end{aligned}
$$

where

$$
\nu_{5}(\ell(2, n)) \geq \min _{1 \leq k \leq 5}\left(\nu_{5}(\ell(1, k))+\nu_{5}(b(k, n)) \geq \min _{1 \leq k \leq 5}\left(\left\lfloor\frac{3 k-2}{4}\right\rfloor+\left\lfloor\frac{3 n-k+2}{4}\right\rfloor\right)=\left\lfloor\frac{3 n+1}{4}\right\rfloor,\right.
$$

since when $k=1,\left\lfloor\frac{3 k-2}{4}\right\rfloor+\left\lfloor\frac{3 n-k+2}{4}\right\rfloor=\left\lfloor\frac{3 n+1}{4}\right\rfloor$, and for $k \geq 2$,

$$
\left\lfloor\frac{3 k-2}{4}\right\rfloor+\left\lfloor\frac{3 n-k+2}{4}\right\rfloor \geq\left\lfloor\frac{3 n+2 k-3}{4}\right\rfloor \geq\left\lfloor\frac{3 n+1}{4}\right\rfloor .
$$

Thus the result holds for $L_{2 \alpha}$ when $\alpha=1$. We proceed by induction. Suppose the result holds for $L_{2 \alpha}$ for a given $\alpha \geq 1$. Then by Lemma 3.7 and equation (3.70) we have

$$
\begin{aligned}
L_{2 \alpha+1} & =U_{A}\left(L_{2 \alpha}\right)=\sum_{n \geq 1} \ell(2 \alpha, n) U_{A}\left(P_{A} t^{n}\right), \\
& =\sum_{n \geq 1} \ell(2 \alpha, n) P_{B} \sum_{k \geq 2} a(n, k) t^{k} \\
& =P_{B} \sum_{n \geq 2} \sum_{k \geq 1} \ell(2 \alpha, k) a(k, n) t^{n} \\
& =P_{B} \sum_{n \geq 2} \ell(2 \alpha+1, n) t^{n},
\end{aligned}
$$


where

$$
\begin{aligned}
\nu_{5}(\ell(2 \alpha+1, n)) & \geq \min _{1 \leq k}\left(\nu_{5}(\ell(2 \alpha, k))+\nu_{5}(a(k, n)) \geq \min _{1 \leq k}\left(\alpha+\left\lfloor\frac{3 k-3}{4}\right\rfloor+\left\lfloor\frac{3 n-k}{4}\right\rfloor\right)\right. \\
& \geq \alpha+1+\left\lfloor\frac{3 n-6}{4}\right\rfloor,
\end{aligned}
$$

since when $k=1,\left\lfloor\frac{3 k-3}{4}\right\rfloor+\left\lfloor\frac{3 n-k}{4}\right\rfloor=1+\left\lfloor\frac{3 n-5}{4}\right\rfloor$, and for $k \geq 2$,

$$
\left\lfloor\frac{3 k-3}{4}\right\rfloor+\left\lfloor\frac{3 n-k}{4}\right\rfloor \geq\left\lfloor\frac{3 n+2 k-6}{4}\right\rfloor \geq 1+\left\lfloor\frac{3 n-6}{4}\right\rfloor .
$$

Thus the result holds for $L_{2 \alpha+1}$. Suppose the result holds for $L_{2 \alpha+1}$ for a given $\alpha \geq 1$. Then again by Lemma 3.7 and equation (3.69) we have

$$
\begin{aligned}
L_{2 \alpha+2} & =U_{B}\left(L_{2 \alpha+1}\right)=\sum_{n \geq 2} \ell(2 \alpha+1, n) U_{B}\left(P_{B} t^{n}\right), \\
& =\sum_{n \geq 2} \ell(2 \alpha+1, n) P_{A} \sum_{k \geq 1} b(n, k) t^{k} \\
& =P_{A} \sum_{n \geq 1} \sum_{k \geq 2} \ell(2 \alpha+1, k) b(k, n) t^{n} \\
& =P_{A} \sum_{n \geq 1} \ell(2 \alpha+2, n) t^{n}
\end{aligned}
$$

where $\ell(2 \alpha+1,1)=0$. Here

$$
\begin{aligned}
\nu_{5}(\ell(2 \alpha+2, n)) & \geq \min _{2 \leq k}\left(\nu_{5}(\ell(2 \alpha+1, k))+\nu_{5}(b(k, n)) \geq \min _{2 \leq k}\left(\alpha+1+\left\lfloor\frac{3 k-6}{4}\right\rfloor+\left\lfloor\frac{3 n-k+2}{4}\right\rfloor\right)\right. \\
& \geq \min _{2 \leq k}\left(\alpha+1+\left\lfloor\frac{3 n+2 k-7}{4}\right\rfloor\right)=\alpha+1+\left\lfloor\frac{3 n-3}{4}\right\rfloor .
\end{aligned}
$$

Thus the result holds for $L_{2 \alpha+2}$, and the result holds in general by induction.

Corollary 3.10. For $\alpha \geq 1$ and all $n \geq 0$ we have

$$
\begin{aligned}
& c_{f}\left(5^{2 \alpha} n+\lambda_{2 \alpha}\right) \equiv 0 \quad\left(\bmod 5^{\alpha}\right), \\
& c_{f}\left(5^{2 \alpha+1} n+\lambda_{2 \alpha+1}\right) \equiv 0 \quad\left(\bmod 5^{\alpha+1}\right) .
\end{aligned}
$$

Proof. The congruences follow immediately from Lemma 3.8 and Theorem 3.9.

In view of (3.66) and Corollary 3.10 we obtain (1.4). This completes the proof of Theorem 1.2.

\section{Further results}

The methods of this paper can be extended to study congruences mod powers of 7 for both the rank and crank parity functions. We describe some of these results, which we will prove in a subsequent paper [CCG21]. Analogous to (3.34) we find that

$$
\sum_{n=0}^{\infty}\left(a_{f}(n / 7)-a_{f}(7 n-2)\right) q^{n}=\frac{J_{7}^{3}}{J_{2}^{2}}\left(\frac{J_{1}^{3} J_{7}^{3}}{J_{2}^{3} J_{14}^{3}}+6 q^{2} \frac{J_{14}^{4} J_{1}^{4}}{J_{2}^{4} J_{7}^{4}}\right)
$$

which leads to the following 
Theorem 4.1. For all $\alpha \geq 3$ and all $n \geq 0$ we have

$$
a_{f}\left(7^{\alpha} n+\delta_{\alpha}\right)-a_{f}\left(7^{\alpha-2} n+\delta_{\alpha-2}\right) \equiv 0 \quad\left(\bmod 7^{\left\lfloor\frac{1}{2}(\alpha-1)\right\rfloor}\right),
$$

where $\delta_{\alpha}$ satisfies $0<\delta_{\alpha}<7^{\alpha}$ and $24 \delta_{\alpha} \equiv 1\left(\bmod 7^{\alpha}\right)$.

As mentioned earlier in Section 1., Karl-Heinz Fricke [Fr13, p.232] independently observed this theorem and related results for Ramanujan's third order mock theta function $\omega(q)$, but without proof.

It turns out that for the crank parity function congruences mod powers of 7 are more difficult. Define the crank parity function

$$
\beta(n)=M_{e}(n)-M_{o}(n),
$$

for all $n \geq 0$. The following is our analog of Choi, Kang and Lovejoy's Theorem 1.1.

Theorem 4.2. For each $\alpha \geq 1$ there is an integral constant $K_{\alpha}$ such that

$$
\beta(49 n-2) \equiv K_{\alpha} \beta(n) \quad\left(\bmod 7^{\alpha}\right), \quad \text { if } 24 n \equiv 1 \quad\left(\bmod 7^{\alpha}\right) .
$$

This gives a weak refinement of Ramanujan's partition congruence modulo powers of 7 :

$$
p(n) \equiv 0 \quad\left(\bmod 7^{\alpha}\right), \quad \text { if } 24 n \equiv 1 \quad\left(\bmod 7^{\left\lfloor\frac{a+2}{2}\right\rfloor}\right) .
$$

This was also proved by Watson [Wa38]. Atkin and O'Brien [AO67] obtained congruences mod powers of 13 for the partition function similar to (4.76).

Acknowledgement. We would like to thank Jonathan Bradley-Thrush for his comments and suggestions. As well we thank Karl-Heinz Fricke for his comments and for sharing some of his recent work. Finally, we thank the referee for corrections and helpful comments.

\section{References}

[AhDu19] Scott Ahlgren and Alexander Dunn, Maass forms and the mock theta function f(q), Math. Ann. 374 (2019), no. 3-4, 1681-1718. MR3985121

[AnGa88] George E. Andrews and F.G. Garvan, Dyson's crank of a partition, Bull. Amer. Math. Soc. (N.S.) 18 (1988), no. 2, 167-171. MR929094

[An84] George E. Andrews, Generalized Frobenius partitions, Mem. Amer. Math. Soc. 49 (1984), no. 301, iv+44. MR743546

[An66] George E. Andrews, On the theorems of Watson and Dragonette for Ramanujan's mock theta functions, Amer. J. Math. 88 (1966), 454-490. MR200258

[AtLe70] A.O.L. Atkin and J. Lehner, Hecke operators on $\Gamma_{0}(m)$, Math. Ann. 185 (1970), 134-160. MR0268123

[AO67] A.O.L. Atkin and J.N. O'Brien, Some properties of $p(n)$ and $c(n)$ modulo powers of 13 , Trans. Amer. Math. Soc. 126 (1967), 442-459. MR214540

[AS-D54] A.O.L. Atkin and P. Swinnerton-Dyer, Some properties of partitions, Proc. London Math. Soc. (3) 4 (1954), 84-106. MR0060535

[Be91] Bruce C. Berndt, Ramanujan's notebooks. Part III, Springer-Verlag, New York, 1991. MR1117903

[Bi89] Anthony J.F. Biagioli, A proof of some identities of Ramanujan using modular forms, Glasgow Math. J. 31 (1989), no. 3, 271-295. MR1021804

[BrOn06] Kathrin Bringmann and Ken Ono, The $f(q)$ mock theta function conjecture and partition ranks, Invent. Math. 165 (2006), no. 2, 243-266. MR2231957

[CCG21] Dandan Chen, Rong Chen, and Frank Garvan, Congruences modulo powers of 7 for the rank and crank parity functions, (2021), in preparation.

[CKL09] Dohoon Choi, Soon-Yi Kang, and Jeremy Lovejoy, Partitions weighted by the parity of the crank, J. Combin. Theory Ser. A 116 (2009), no. 5, 1034-1046. MR2522417

[CKP09] Bumkyu Cho, Ja Kyung Koo, and Yoon Kyung Park, Arithmetic of the Ramanujan-Göllnitz-Gordon continued fraction, J. Number Theory 129 (2009), no. 4, 922-947. MR2499414 
[ChLa04] Kok Seng Chua and Mong Lung Lang, Congruence subgroups associated to the monster, Experiment. Math. 13 (2004), no. 3, 343-360. MR2103332

[Dr52] Leila A. Dragonette, Some asymptotic formulae for the mock theta series of Ramanujan, Trans. Amer. Math. Soc. 72 (1952), 474-500. MR49927

[Dy44] F.J. Dyson, Some guesses in the theory of partitions, Eureka (1944), no. 8, 10-15. MR3077150

[FrGa19] Jie Frye and Frank Garvan, Automatic proof of theta-function identities, Elliptic integrals, elliptic functions and modular forms in quantum field theory, Texts Monogr. Symbol. Comput., Springer, Cham, 2019, pp. 195-258. MR3889559

[Fr13] Karl-Heinz Fricke, Analytische und p-adische Aspekte von klassischen und Mock-Modulformen, Ph.D. thesis, Rheinischen Friedrich-Wilhelms-Universität Bonn, 2013, pp.307. https://d-nb.info/1045276588/34

[Ga88] F.G. Garvan, New combinatorial interpretations of Ramanujan's partition congruences mod 5, 7 and 11 , Trans. Amer. Math. Soc. 305 (1988), no. 1, 47-77. MR920146

[GoHu81] B. Gordon and K. Hughes, Ramanujan congruences for $q(n)$, Analytic number theory (Philadelphia, Pa., 1980), Lecture Notes in Math., vol. 899, Springer, Berlin-New York, 1981, pp. 333-359. MR654539

[HiHu81] Michael D. Hirschhorn and David C. Hunt, A simple proof of the Ramanujan conjecture for powers of 5, J. Reine Angew. Math. 326 (1981), 1-17. MR622342

[Li75] Gérard Ligozat, Courbes modulaires de genre 1, Société Mathématique de France, Paris, 1975, Bull. Soc. Math. France, Mém. 43, Supplément au Bull. Soc. Math. France Tome 103, no. 3. MR0417060

[Ma09] Robert S. Maier, On rationally parametrized modular equations, J. Ramanujan Math. Soc. 24 (2009), no. 1, 1-73. MR2514149

[Mao13] Renrong Mao, Ranks of partitions modulo 10, J. Number Theory 133 (2013), no. 11, 3678-3702. MR3084295

[Ne59] Morris Newman, Construction and application of a class of modular functions. II, Proc. London Math. Soc. (3) 9 (1959), 373-387. MR0107629

[PaRa12] Peter Paule and Cristian-Silviu Radu, The Andrews-Sellers family of partition congruences, Adv. Math. 230 (2012), no. 3, 819-838. MR2921161

[Ra77] Robert A. Rankin, Modular forms and functions, Cambridge University Press, Cambridge, 1977. MR0498390

[Ro94] Sinai Robins, Generalized Dedekind $\eta$-products, The Rademacher legacy to mathematics (University Park, PA, 1992), Contemp. Math., vol. 166, Amer. Math. Soc., Providence, RI, 1994, pp. 119-128. MR1284055

[Se94] James Sellers, Congruences involving F-partition functions, Internat. J. Math. Math. Sci. 17 (1994), no. 1, 187-188. MR1255240

[Wa36] G.N. Watson, The Final Problem : An Account of the Mock Theta Functions, J. London Math. Soc. 11 (1936), no. 1, 55-80. MR1573993

[Wa38] G.N. Watson, Ramanujans Vermutung über Zerfällungszahlen, J. Reine Angew. Math. 179 (1938), 97-128. MR1581588

\section{Dandan Chen}

Department of Mathematics

Shanghai University, Shanghai

People's Republic of China

e-mail: mathcdd@shu.edu.cn

\section{Rong Chen}

School of Mathematical Sciences

Tongji University, Shanghai

People's Republic of China

e-mail: rongchen20@tongji.edu.cn

\section{Frank Garvan}

Department of Mathematics

University of Florida, Gainesville

FL 32611-8105, USA

e-mail: fgarvan@ufl.edu 